\title{
Faster Coroutine Pipelines: A Reconstruction
}

\author{
Ruben P. Pieters ${ }^{凶[0000-0003-0537-9403]}$ and Tom Schrijvers ${ }^{[0000-0001-8771-5559]}$ \\ KU Leuven, 3001 Leuven, Belgium \\ \{ruben.pieters, tom.schrijvers\}@cs.kuleuven.be
}

\begin{abstract}
Spivey has recently presented a novel functional representation that supports the efficient composition, or merging, of coroutine pipelines for processing streams of data. This representation was inspired by Shivers and Might's three-continuation approach and is shown to be equivalent to a simple yet inefficient executable specification. Unfortunately, neither Shivers and Might's original work nor the equivalence proof sheds much light on the underlying principles allowing the derivation of this efficient representation from its specification.

This paper gives the missing insight by reconstructing a systematic derivation in terms of known transformation steps from the simple specification to the efficient representation. This derivation sheds light on the limitations of the representation and on its applicability to other settings. In particular, it has enabled us to obtain a similar representation for pipes featuring two-way communication, similar to the Haskell pipes library. Our benchmarks confirm that this two-way representation retains the same improved performance characteristics.
\end{abstract}

Keywords: Stream Processing - Structured Recursion - Algebra.

\section{Introduction}

Coroutine pipelines provide a compositional approach to processing streams of data that is both efficient in time and space, thanks to a targeted form of lazy evaluation interacting well with side-effects like I/O. Two prominent Haskell libraries for coroutine pipelines are pipes [?] and conduit [?]. Common to both libraries is their representation of pipelines by an algebraic data type (ADT).

Spivey [?] has recently presented a novel Haskell representation that is entirely function-based. His representation is an adaptation of Shivers and Might's earlier three-continuation representation [?] and exhibits a very efficient merge operation for connecting pipes.

Spivey proves that his representation is equivalent to a simple ADT-based specification. Yet, neither his proof nor Shivers and Might's explanation sheds much light on the underlying principles used to come up with the efficient representation. This makes it difficult to adapt the representation to other settings.

This paper remedies the situation by systematically deriving the efficient function-based representation from the simple, but inefficient ADT-based representation. Our derivation consists of known transformations and constructions that are centered around folds with appropriate algebras. Our derivation clarifies 
the limitations of the efficient representation, and enables us to derive a similarly efficient represenation for the two-way pipes featured in the pipes library.

The specific contributions of this paper are:

- We present a systematic derivation of Spivey's efficient representation starting from a simple executable specification. Our derivation only consists of known transformations, most of which concern structural recursion with folds and algebras. It also explains why the efficient representation only supports the merging of "never-returning" pipes.

- We apply our derivation to a more general definition of pipes used by the pipes library, where the communication between adjacent pipes is bidirectional rather than unidirectional.

- Our benchmarks demonstrate that the merge operator for the bidirectional three-continuation approach improves upon the pipes library's performance.

The rest of this paper is organized as follows. Section ?? briefly introduces both the ADT pipes encoding and the three-continuation approach. Section ?? derives the fast merging operation for a simplified setting. Section ?? derives the fast merging operation for the original pipe setting. Section ?? extends Spivey's approach with the bidirectional pipes operations. Section ?? presents the results of the primes benchmark by Spivey, on the approaches discussed in this paper. Section ?? discusses related work and Section ?? concludes this paper. The appendix is included in the extended version ${ }^{1}$.

\section{Motivation}

This section introduces the ADT pipes encoding and then contrasts it with the three-continuation encoding. This serves as both a background introduction and a motivation for a better understanding of the relation between both encodings.

\section{$2.1 \quad$ Pipes}

We start with a unidirectional version of the pipes library. A unidirectional pipe can receive $i$ values, output $o$ values and return $a$ values. On the other hand, a bidirectional pipe additionally carries an output value when receiving values and an input value when outputting values. We represent a unidirectional pipe as an abstract syntax tree where each node is an input, output or return operation. This is expressed in Haskell with the following ADT.

$$
\begin{aligned}
\text { data Pipe } i \text { o a } & =\text { Input }(i \rightarrow \text { Pipe } i \text { o a }) \\
& \mid \text { Output o (Pipe } i \text { o a }) \\
& \mid \text { Return a }
\end{aligned}
$$

This datatype exhibits a monadic structure where the bind operation ( $\gg)$ grafts one syntax tree onto another.

\footnotetext{
${ }^{1}$ http://www.cs.kuleuven.be/publicaties/rapporten/cw/CW715.abs.html
} 


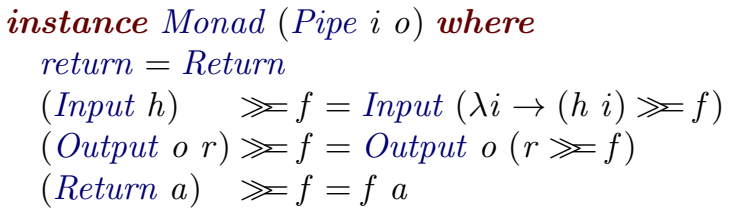

We define the basic components: input $_{P}$ : a pipe returning its received input, output $_{P}$ : a pipe outputting a set value and $\operatorname{return}_{P}$ : a pipe returning a set value.

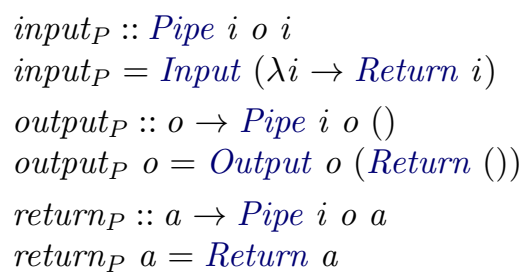

The bind operation assembles these componenents into larger pipes. For example doubler $_{P}$, a pipe which repeatedly takes its input, multiplies it by two and continually outputs this new value.

$$
\begin{aligned}
& \text { doubler }_{P}:: \text { Pipe Int Int a } \\
& \text { doubler }_{P}=\text { do } i \leftarrow \text { input }_{P} ; \text { output }_{P}(i * 2) ; \text { doubler }_{P}
\end{aligned}
$$

Another essential way of combining pipes is merging them. This connects the outputs of the upstream to the inputs of the downstream. In the implementation, merge $_{P L}$ performs a case analysis on the downstream $q$ : if it is trying to output then that is kept and we keep searching, if it finds an input then we call merge $e_{P R}$ on the wrapped continuation and the upstream. Then, in merge $_{P R}$ we similarly scan the upstream for an output operation, keeping any input operations. If an output operation is found, the output value is passed to the continuation and the merging process starts again. If at any point we see a return, then the merge finishes with this resulting return value. The implementation is given below.

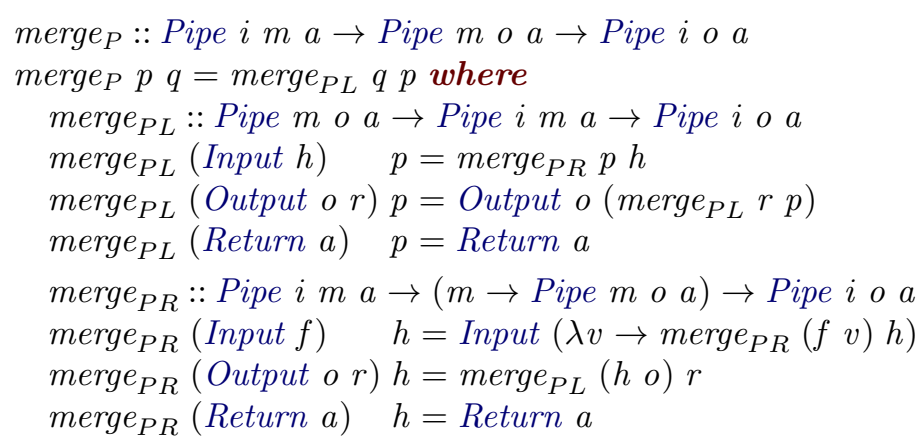

The merge operator enables expressing the merge of doubler $_{P}$ with itself. In this example the left doubler $_{P}$ is the upstream and the right doubler $_{P}$ is the 
downstream. The result of this merge is a pipe which outputs the quadruple of its incoming values.

$$
\begin{aligned}
& \text { quadrupler }_{P}:: \text { Pipe Int Int a } \\
& \text { quadrupler }_{P}=\text { doubler }_{P} \text { ' } \text { merge }_{P} \text { ' } \text { doubler }_{P}
\end{aligned}
$$

We can run a pipe by interpreting it to $I O$.

$$
\begin{aligned}
& \text { toIO }_{P}::(\text { Read } i, \text { Show o }) \Rightarrow \text { Pipe } i \text { o a } \rightarrow I O \text { a } \\
& \text { toIO }_{P}(\text { Input } f) \quad=\text { do } i \leftarrow \text { readLn; } \text { toIO }_{P}(f i) \\
& \text { toIO }{ }_{P}(\text { Output o } r)=\text { do putStrLn ("out: " + show o); toI } O_{P} r \\
& \text { toIO } O_{P}(\text { Return } a)=\text { return } a
\end{aligned}
$$

An example where we input 10 , receive 40 and then exit, is shown below.

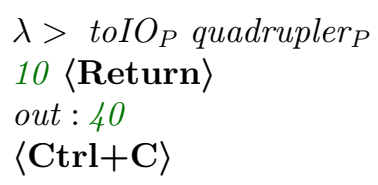

\subsection{Three-Continuation Approach}

The function merge $_{P}$ is suboptimal because it has to recursively scan a pipe for an operation of interest while copying the other operation. When several merges are piled up this leads to repeated scanning and copying of the same operations.

Spivey has introduced ContPipe, a different pipe representation which enables a faster merge implementation [?]. It featuresthree continuations, one for each constructor. The first continuation ( $a \rightarrow$ Result $i$ o) represents the return constructor. The next two continuations, InCont $i$ and OutCont $o$ as part of Result $i$ o, represent the input and output constructor respectively.

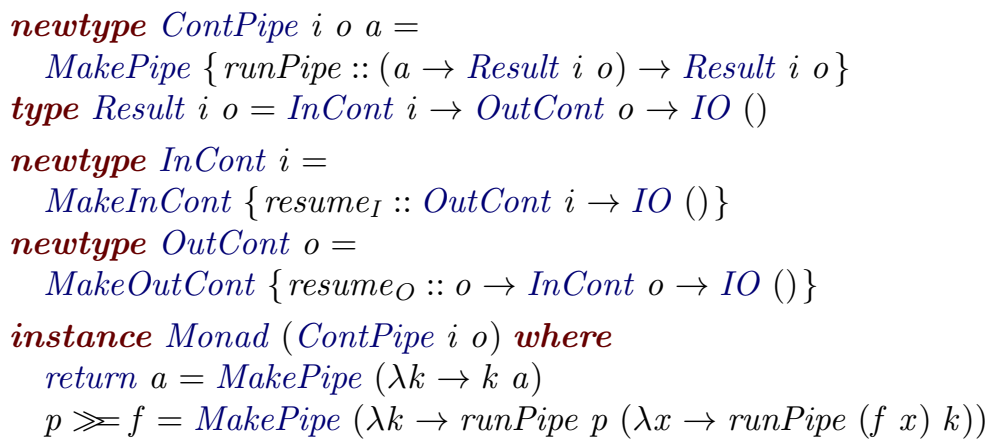

In the following definitions for the basic pipe components the continuation $k$ is the return constructor - we give it a value and the input and output constructors and receive a pipe. The continuations $k_{i}$ and $k_{o}$ are the input and output constructors, we resume them with the newtype unwrapper and the continuations are refreshed once they have been used. 


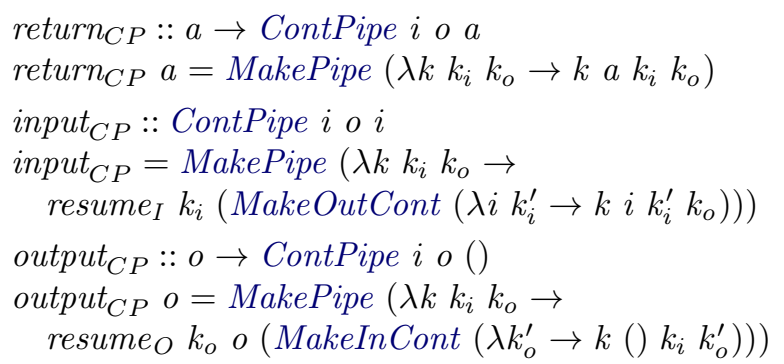

We can use the Monad instance for ContPipe to compose pipes with $\boldsymbol{d} \boldsymbol{o}$-notation, similar to Pipe.

$$
\begin{aligned}
& \text { doubler }_{C P}:: \text { ContPipe Int Int a } \\
& \text { doubler }_{C P}=\text { do } i \leftarrow \text { input }_{C P} ; \text { output }_{C P}(i * 2) ; \text { doubler }_{C P}
\end{aligned}
$$

We can also interpret ContPipe to IO.

$$
\begin{aligned}
& \text { toIO }_{C P}::(\text { Read } i, \text { Show o }) \Rightarrow \text { ContPipe } i \text { o }() \rightarrow I O() \\
& \text { toIO }_{C P} p=\text { runPipe } p(\lambda()--\rightarrow \text { return }()) k_{i} k_{o} \text { where } \\
& k_{i}=\text { MakeInCont }\left(\lambda k_{o} \rightarrow \text { do } x \leftarrow \text { readLn; resume } k_{o} x k_{i}\right) \\
& k_{o}=\text { MakeOutCont }\left(\lambda o k_{i} \rightarrow\right. \\
& \left.\quad \text { do putStrLn }(\text { "out: " }+ \text { show o }) ; \text { resume }_{I} k_{i} k_{o}\right)
\end{aligned}
$$

The merge function for ContPipe is defined as:

$$
\begin{aligned}
& \text { merge }_{C P} p q=\text { MakePipe }\left(\lambda k k_{i} k_{o} \rightarrow\right. \\
& \left.\quad \text { runPipe q err }\left(\text { MakeInCont }\left(\lambda k_{o}^{\prime} \rightarrow \text { runPipe } p \text { err } k_{i} k_{o}^{\prime}\right)\right) k_{o}\right) \\
& \text { where err = error "terminated" }
\end{aligned}
$$

With the merge definition we are able to create the quadrupler pipe as before. Running $t_{0 I O_{C P}}$ quadrupler $_{C P}$ results an identical scenario to the Pipe scenario from the previous section.

$$
\begin{aligned}
& \text { quadrupler }_{C P}:: \text { ContPipe Int Int a } \\
& \text { quadrupler }_{C P}=\text { doubler }_{C P} \text { ' } \text { merge }_{C P} \text { ' } \text { doubler }_{C P}
\end{aligned}
$$

While Spivey has demonstrated the remarkable performance advantage of this merge operator, he sheds little light on the origin or underlying principles of the related encoding. The remainder of this paper provides this missing insight by deriving Spivey's efficient ContPipe representation from the ADT-style Pipe by means of well-known principles. The aim is to improve understanding of the applicability and limitations of the techniques used.

\section{Fast Merging for One-Sided Pipes}

To offer a firmer grip on the problem, this section considers a simplified setting where pipes are one-sided, either only producing or only consuming data. For example, the doubler component can not be defined in this setting. The simplified setting gives a more straightforward path to the fast merging approach, which we generalize back to regular 'mixed' pipes in Section ??. 


\subsection{One-Sided Pipes}

In the simplified setting pipes are either pure Producers or pure Consumers. A Producer only outputs values, while a Consumer only receives them.

data Producer $o=$ Producer $o($ Producer $o)$

data Consumer $i=$ Consumer $(i \rightarrow$ Consumer $i)$

If we specialize merge $_{P}$ for a Consumer and a Producer, we get:

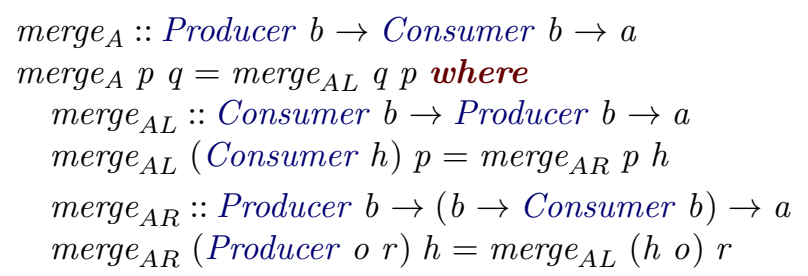

\subsection{Mutual Recursion Elimination}

The two auxiliary functions merge $_{A L}$ and merge $_{A R}$ turn respectively a producer and a consumer into the result of type $a$ by means of an additional parameter, which is respectively of type (Producer $b)$ and $(b \rightarrow$ Consumer $b)$. To highlight these parameters, we introduce type synonyms for them.

type ProdPar' $b=$ Producer $b$

type ConsPar' $b=b \rightarrow$ Consumer $b$

Now we refactor merge $_{A L}$ and merge $_{A R}$ with respect to their additional parameter in a way that removes the term-level mutual recusion between them. Consider merge $_{A L}$ which does not use its parameter $p$ directly, but only its interpretation by function $\operatorname{merge}_{A R}$. We refactor this code to a form where merge $_{A R}$ has already been applied to $p$ before it is passed to merge $e_{A L}$. This adapted merge $e_{A L}$ would then have type Consumer $b \rightarrow\left(\right.$ ConsPar $\left.^{\prime} b \rightarrow a\right) \rightarrow a$. At the same time we apply a similar transformation to merge $_{A R}$, moving the application of merge $_{A L}$ to $h$ out of it. This yields infinite types for the two new parameters, which Haskell only accepts if we wrap them in newtypes.

newtype ProdPar $b a=$ ProdPar (ConsPar $b a \rightarrow a$ )

newtype ConsPar $b a=$ ConsPar $(b \rightarrow$ ProdPar $b a \rightarrow a)$

The merge is then defined by appropriately placing newtype (un-)wrappers.

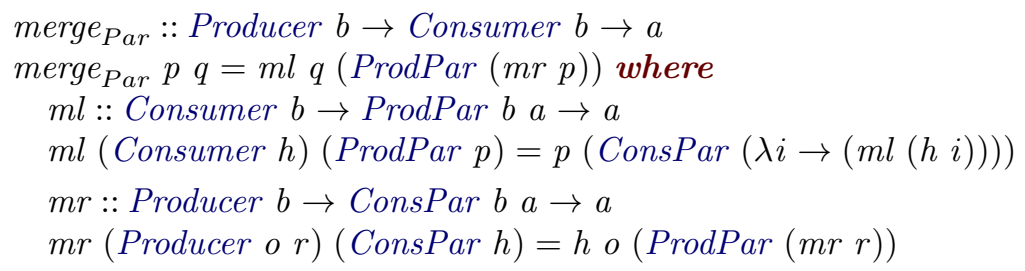

Note that we can recover Spivey's InCont $i$ and OutCont $o$ by instantiating the type parameter a to IO () in ProdPar $i$ a and ConsPar o a respectively. 


\subsection{Structural Recursion with Fold}

Due to the removal of the term-level mutual recursion in $m l$ and $m r$, they are easily adapted to their structurally recursive form. By isolating the work done in each recursive step, we obtain $a g_{L}$ and $a g_{R}$.

$$
\begin{aligned}
& {\text { type } \text { Carrier }_{L} i a=\operatorname{ProdPar} i a \rightarrow a}_{a} \\
& \operatorname{alg}_{L}::\left(i \rightarrow \text { Carrier }_{L} i a\right) \rightarrow \text { Carrier }_{L} i a \\
& \operatorname{alg}_{L} f=\lambda(\text { ProdPar prod }) \rightarrow \operatorname{prod}(\text { ConsPar } f)
\end{aligned}
$$

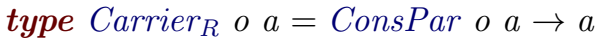

$$
\begin{aligned}
& \operatorname{alg}_{R}:: o \rightarrow \text { Carrier }_{R} \text { o } a \rightarrow \text { Carrier }_{R} \text { o a } \\
& \operatorname{alg}_{R} \text { o prod }=\lambda(\text { ConsPar cons }) \rightarrow \text { cons o }(\text { ProdPar prod })
\end{aligned}
$$

The functions $a l g_{L}$ and $a l g_{R}$ are now in a form known as algebras. Algebras are a combination of a carrier $r$, the type of the resulting value, and an action of type $f r \rightarrow r$. This action denotes the computation performed at each node of the recursive datatype, for which the functor $f$ determines the shape of its nodes. We omit the carrier type if it is clear from the context and simply refer to an algebra by its action.

The structural recursion schemes, or folds, for Consumer and Producer take algebras of the form $(i \rightarrow r) \rightarrow r$ and $o \rightarrow r \rightarrow r$. Their definitions are:

$$
\begin{aligned}
& \text { fold }_{P}::(o \rightarrow r \rightarrow r) \rightarrow \text { Producer } o \rightarrow r \\
& \text { fold }_{P} \text { alg }(\text { Producer o } r)=\text { alg o }\left(\text { fold }_{P} \text { alg } r\right) \\
& \text { fold }_{C}::((i \rightarrow r) \rightarrow r) \rightarrow \text { Consumer } i \rightarrow r \\
& \text { fold }_{C} \text { alg }(\text { Consumer } h)=\text { alg }\left(\lambda i \rightarrow \text { fold }_{C} \text { alg }(h i)\right)
\end{aligned}
$$

An example use of folds is an interpretation to $I O$ by supplying the inputs for a consumer or printing the outputs of a producer.

$$
\begin{aligned}
& \text { type CarrierCons } \text { IO } i=I O() \\
& \text { consume }_{I O}:: \text { Read } i \Rightarrow \text { Consumer } i \rightarrow I O() \\
& \text { consume }_{I O}=\text { fold }_{C} \text { alg where } \\
& \text { alg }:: \text { Read } i \Rightarrow\left(i \rightarrow \text { CarrierCons }_{I O} i\right) \rightarrow \text { CarrierCons }_{I O} i \\
& \operatorname{alg} f=\text { do } x \leftarrow \operatorname{readLn} ; f x \\
& \text { type CarrierProd } \text { IO }_{\text {o }}=I O() \\
& \text { produce }_{I O}:: \text { Show o } \Rightarrow \text { Producer } o \rightarrow I O() \\
& \text { produce }_{I O}=\text { fold }_{P} \text { alg where } \\
& \text { alg :: Show o } \Rightarrow \text { o } \rightarrow \text { CarrierProd }_{I O} o \rightarrow \text { CarrierProd }_{I O} o \\
& \text { alg o } p=\text { do print } o ; p
\end{aligned}
$$

Another example is expressing merge $e_{\text {Par }}$ with folds using $\operatorname{alg}_{L}$ and $a g_{R}$.

$$
\begin{aligned}
& \text { merge }_{\text {fold }}:: \text { Producer } x \rightarrow \text { Consumer } x \rightarrow a \\
& \text { merge }_{\text {fold }} p q=\text { fold }_{C} \text { alg }_{L} q\left(\text { ProdPar }\left(\text { fold }_{P} \operatorname{alg}_{R} p\right)\right)
\end{aligned}
$$




\subsection{A Short-Cut to a Merge-Friendly Representation}

Instead of directly defining a Consumer or Producer value in terms of the data constructors of the respective types, we can also do it in a more roundabout way by abstracting over the constructor occurrences - this is known as build form. The build function then instantiates the abstracted constructors with the actual constructors; for Consumer and Producer they are:

$$
\begin{aligned}
& \text { build }_{C}::(\forall r .((i \rightarrow r) \rightarrow r) \rightarrow r) \rightarrow \text { Consumer } i \\
& \text { build }_{C} g=g \text { Consumer } \\
& \text { build }_{P}::(\forall r .(o \rightarrow r \rightarrow r) \rightarrow r) \rightarrow \text { Producer o } \\
& \text { build }_{P} g=g \text { Producer }
\end{aligned}
$$

For instance,

$$
\begin{aligned}
& \text { prodFrom }:: \text { Integer } \rightarrow \text { Producer Integer } \\
& \text { prodFrom } n=\text { Producer } n(\text { prodFrom }(n+1))
\end{aligned}
$$

can be written as:

$$
\begin{aligned}
& \text { prodFrom } n=\text { build }_{P}\left(\text { prodFrom }^{\prime} n\right) \text { where } \\
& \quad \text { prodFrom' }:: \text { Integer } \rightarrow(\forall r .(\text { Integer } \rightarrow r \rightarrow r) \rightarrow r) \\
& \text { prodFrom }^{\prime} n p=\text { go } n \text { where go } n=p n(\text { go }(n+1))
\end{aligned}
$$

The motivation for these build functions is use of the fold/build fusion rule, a special form of short-cut fusion [?]. This rule can be applied when a fold directly follows a build, specifically for Consumer and Producer these fusion rules are:

$$
\begin{aligned}
& \text { fold }_{C} \text { alg }\left(\text { build }_{C} \text { cons }\right)=\text { cons alg } \\
& \text { fold }_{P} \text { alg }\left(\text { build }_{P} \text { prod }\right)=\text { prod alg }
\end{aligned}
$$

In other words, instead of first building an ADT representation and then folding it to its result, we can directly create the result of the fold. This readily applies to the two folds in merge $_{\text {fold }}$. We can directly represent consumers and producers in terms of the carrier types of those two folds,

$$
\begin{aligned}
& \text { type Consumer }_{\text {Alt }} i=\forall \text { a.Carrier } L \text { ia } a \quad-\forall \text { a.ProdPar } i a \rightarrow a \\
& \text { type Producer }_{A l t} \quad o=\forall a . \text { Carrier }_{R} \text { o a } \quad-\forall \text { a.ConsPar o } a \rightarrow a
\end{aligned}
$$

using their algebras as constructors:

$$
\begin{aligned}
& \text { input }_{\text {Alt }}::\left(i \rightarrow \text { Consumer }_{A l t} i\right) \rightarrow \text { Consumer }_{A l t} i \\
& \text { input }_{A l t}=\text { alg }_{L} \\
& \text { output }_{A l t}:: o \rightarrow \text { Producer }_{A l t} \text { o } \rightarrow \text { Producer }_{A l t} O \\
& \text { output }_{\text {Alt }}=\text { alg }_{R}
\end{aligned}
$$

For instance, after fold/build fusion prodFrom becomes: 


$$
\begin{aligned}
& \operatorname{prodFrom}_{\text {Alt }}:: \text { Integer } \rightarrow \text { Producer }_{\text {Alt Integer }} \\
& \text { prodFrom }_{\text {Alt }} n=\text { output }_{A l t} n\left(\text { prodFrom }_{\text {Alt }}(n+1)\right)
\end{aligned}
$$

The merge function for the alternate representations Producer $_{\text {Alt }}$ and Consumer $_{\text {Alt }}$ then becomes an almost trivial operation.

$$
\begin{aligned}
& \text { merge }_{\text {Alt }}:: \text { Producer }_{\text {Alt }} b \rightarrow \text { Consumer }_{\text {Alt }} b \rightarrow a \\
& \text { merge }_{\text {Alt }} p q=q(\text { ProdPar } p)
\end{aligned}
$$

\subsection{A Not So Special Representation}

This merge-friendly representations of producers and consumers are not just specializations; they are in fact isomorphic to the originals. The inverses of $m l$ and $m r$ to complete the isomorphism are given by $m l^{-1}$ and $m r^{-1}$. The proof is included in the appendix.

$$
\begin{aligned}
& m l^{-1}:: \text { Consumer }_{A l t} i \rightarrow \text { Consumer } i \\
& m l^{-1} f=f(\text { ProdPar } h) \text { where } \\
& h:: \text { ConsPar } i(\text { Consumer } i) \rightarrow \text { Consumer } i \\
& \quad h(\text { ConsPar } f)=\text { Consumer }(\lambda x \rightarrow f x(\text { ProdPar } h)) \\
& m r^{-1}:: \text { Producer } A l t \text { o } \rightarrow \text { Producer o } \\
& m r^{-1} f=f(\text { ConsPar }(\lambda x p \rightarrow \text { Producer } x(h p))) \text { where } \\
& \quad h:: \text { ProdPar o }(\text { Producer } o) \rightarrow \text { Producer o } \\
& \quad h(\text { ProdPar } f)=f(\text { ConsPar }(\lambda x p \rightarrow \text { Producer } x(h p)))
\end{aligned}
$$

Hence, we can also fold with other algebras by transforming the merge-friendly representation back to the ADT, and then folding over that.

$$
\begin{aligned}
& \text { fold }_{P_{A l t}}::(o \rightarrow a \rightarrow a) \rightarrow \text { Producer }_{\text {Alt }} \text { o } \rightarrow a \\
& \text { fold }_{P_{A l t}} \text { alg rep }=\text { fold }_{P} \text { alg }\left(\text { mr }^{-1} \text { rep }\right) \\
& \text { fold }_{C_{A l t}}::((i \rightarrow a) \rightarrow a) \rightarrow \text { Consumer }_{A l t} i \rightarrow a \\
& \text { fold }_{C_{A l t}} \text { alg rep }=\text { fold }_{C} \text { alg }\left(\text { ml }^{-1} \text { rep }\right)
\end{aligned}
$$

Of course, these definitions are wasteful because they create intermediate datatypes. However, by performing fold/build fusion we obtain their fused versions:

$$
\begin{aligned}
& \text { fold }_{P_{A l t}} \text { alg rep }=\text { rep }(\text { ConsPar }(\lambda x p \rightarrow \text { alg } x(h p))) \text { where } \\
& h(\text { ProdPar } f)=f(\text { ConsPar }(\lambda x p \rightarrow \text { alg } x(h p))) \\
& \text { fold }_{C_{A l t} \text { alg rep }=\operatorname{rep}(\text { ProdPar } h) \text { where }} \\
& h(\text { ConsPar } f)=\operatorname{alg}(\lambda x \rightarrow f x(\text { ProdPar } h))
\end{aligned}
$$

\section{Return to Two-Sided Pipes}

The previous section has derived an efficient approach for simplified Consumer and Producer pipes. This section extends that approach to proper Pipes in two steps, first supporting both input and output operations, and then also a return. 


\subsection{Pipe of No Return}

Let us consider pipes with both input and output operations, but no return.

$$
\begin{array}{r}
\text { data } \text { Pipe }_{\infty} i o=\text { Input }_{\infty}\left(i \rightarrow \text { Pipe }_{\infty} i o\right) \\
\mid \text { Output }_{\infty} \text { o }\left(\text { Pipe }_{\infty} i o\right)
\end{array}
$$

We can fold over these pipes by providing algebras for both the input and output operation, agreeing on the carrier type $a$.

$$
\begin{aligned}
& \text { foldPipe }_{\infty}:: \text { Pipe }_{\infty} i o \rightarrow((i \rightarrow a) \rightarrow a) \rightarrow(o \rightarrow a \rightarrow a) \rightarrow a \\
& \text { foldPipe }_{\infty} \text { p inAlg outAlg }=\text { go p where } \\
& \quad \text { go }\left(\text { Input } t_{\infty} p\right)=\text { inAlg }(\lambda i \rightarrow \text { go }(p i)) \\
& \text { go }\left(\text { Output } t_{\infty} \text { o } p\right)=\text { outAlg } \text { o }(\text { go } p)
\end{aligned}
$$

To merge these pipes, we use $a g_{L}$ and $a l g_{R}$ developed in the previous section. There is only one snag: the two algebras do not agree on the carrier type. The carrier types were the alternate representations Consumer $_{\text {Alt }}$ and Producer $_{\text {Alt }}$.

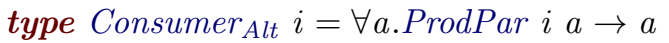

$$
\begin{aligned}
& \text { type Producer }_{A l t} \quad o=\forall \text { a.ConsPar o } a \rightarrow a
\end{aligned}
$$

We reconcile these two carrier types by observing that both are functions with a common result type, but different parameter types. A combination of both is a function taking both parameter types as input.

$$
\text { type Result }_{R} \text { i } o=\forall \text { a.ConsPar o } a \rightarrow \text { ProdPar } i a \rightarrow a
$$

The algebra actions are easily adapted to the additional parameter. They simply pass it on to the recursive positions without using it themselves.

$$
\begin{aligned}
& \text { input }_{\text {Result }_{R}}::\left(i \rightarrow \text { Result }_{R} \text { i o }\right) \rightarrow \text { Result }_{R} \text { i o } \\
& \text { input } \left._{\text {Result }_{R}} f=\lambda \text { cons (ProdPar prod }\right) \rightarrow \\
& \operatorname{prod}\left(\text { ConsPar }\left(\lambda i \text { prod }^{\prime} \rightarrow f i \text { cons prod }{ }^{\prime}\right)\right) \\
& \text { output }_{\text {Result }_{R}}:: o \rightarrow \text { Result }_{R} i o \rightarrow \text { Result }_{R} i o \\
& \text { output }_{\text {Result }_{R}} \text { o result }=\lambda(\text { ConsPar cons }) \text { prod } \rightarrow \\
& \text { cons o (ProdPar } \left.\left(\lambda \text { cons }^{\prime} \rightarrow \text { result cons }{ }^{\prime} \text { prod }\right)\right)
\end{aligned}
$$

Like before, we can avoid the algebraic datatype Pipe $_{\infty}$ and directly work with Result $_{R}$ using the algebras as constructor functions.

Finally, we can use the one-sided merge function from the previous section to merge the output side of a Result ${ }_{R} i m$ pipe with the input side of a Result ${ }_{R} m o$ pipe. Because we defer the interpretation of the $i$ and $o$ sides of the respective pipes, this one-sided merge does not yield a result of type $a$, but rather one of type ConsPar o $a \rightarrow$ ProdPar $i a \rightarrow a$. In other words, the merge of the two pipes yields a Result $_{R} i o$ pipe.

$$
\begin{aligned}
& \text { merge }_{\text {Result }_{R}}:: \text { Result }_{R} i m \rightarrow \text { Result }_{R} m o \rightarrow \text { Result }_{R} i o \\
& \text { merge }_{\text {Result }_{R}} \text { p } q=\lambda \text { cons }_{o} \text { prod }_{i} \rightarrow \\
& \quad \text { let } q^{\prime}=q \text { cons }_{o} \\
& \quad p^{\prime}=\text { flip prod }_{i} \\
& \quad \text { in } q^{\prime}\left(\text { ProdPar }^{\prime}\right)
\end{aligned}
$$




\subsection{Return to return}

Finally, we reobtain return and the monadic structure of pipes in a slightly unusual way, by means of the continuation monad.

$$
\begin{aligned}
& \text { newtype Cont } r a=\text { Cont }\{\text { runCont }::(a \rightarrow r) \rightarrow r\} \\
& \text { instance Monad }(\text { Cont } r) \text { where } \\
& \quad \text { return } x=\text { Cont }(\lambda k \rightarrow k x) \\
& \quad p \gg f=\text { Cont }(\lambda k \rightarrow \text { runCont } p(\lambda x \rightarrow \text { runCont }(f x) k))
\end{aligned}
$$

If we specialize the result type $r$ to $\operatorname{Result}_{R} i o$, we get:

$$
\text { newtype ContP } i \text { o a }=\operatorname{ContP}\left(\left(a \rightarrow \operatorname{Result}_{R} i o\right) \rightarrow \operatorname{Result}_{R} i o\right)
$$

The merge function for ContP is implemented in terms of merge $_{\text {Result }_{R}}$.

$$
\begin{aligned}
& \text { merge }_{\text {Cont }}:: \text { ContP } i m \text { Void } \rightarrow \text { ContP } m \text { o Void } \rightarrow \text { ContP } i \text { o a } \\
& \text { merge }_{\text {Cont }}(\text { ContP p })(\text { ContP } q)=\text { ContP }(\lambda k \rightarrow \\
& \text { merge } \left._{\text {Result }_{R}}(p \text { absurd })(q \text { absurd })\right)
\end{aligned}
$$

However, there is an issue: before merge $_{\text {Result }_{R}}$ can merge the two pipes, their continuations (the interpretations of the return constructor) must be supplied. Yet, the resulting pipe's continuation type $k$ does not match that of either the upstream or downstream pipe. Thus we are stuck, unless we assume what we have been all along: that the two pipes are infinite. Indeed, in that case it does not matter that we don't have a continuation for them, as their continuation is never reached anyway. In short, merge $_{\text {Cont }}$ only works for never-returning pipes, which we signal with the return type Void, only inhabited by $\perp$.

\subsection{Specialization for $I O$}

To get exactly Spivey's representation, we instantiate the polymorphic type variable $a$ in Result $_{R} i$ o to $I O()$, which yields:

$$
\text { type Result } i \text { o }=\text { InCont } i \rightarrow \text { OutCont } o \rightarrow \text { IO () }
$$

We can rewrite this type as a monad transformer stack, using two reader monad transformers for the two parameters.

$$
\begin{aligned}
& \text { newtype ReaderT } r m a=\text { Reader } T \text { \{runReaderT }:: r \rightarrow m a\} \\
& \text { type Result' } i \text { o ReaderT (InCont } i)(\text { ReaderT (OutCont o) IO) () }
\end{aligned}
$$

Similarly, ContPipe $i$ o a can be written with a transformer stack by adding a Cont $T$ layer, since Cont $(m r)$ is equal to Cont $T r m$ for any monad $m$.

$$
\begin{aligned}
& \text { newtype Cont Tr } m \text { a }=\text { Cont } T\{\operatorname{runCont} T::(a \rightarrow m r) \rightarrow m r\} \\
& \text { type ContPipe' } \text { i o a }= \\
& \text { Cont T }() \text { (ReaderT (InCont } i)(\text { ReaderT (OutCont o) IO })) a
\end{aligned}
$$


This transformer stack view enables two additional useful operations: aborting the pipe and embedding an $I O$ action. Both are specializations of generic functionality from the continuation monad transformer: abort and lift ${ }_{\text {Cont } T}$.

$$
\begin{aligned}
& \text { abort : } \mathrm{m} r \rightarrow \text { ContT } r \mathrm{ma} \\
& \text { abort } r=\operatorname{Cont} T(\lambda k \rightarrow r) \\
& \text { lift }_{\text {Cont } T}:: \text { Monad } m \Rightarrow m a \rightarrow \text { ContT } r m a \\
& \text { lift }_{\text {Cont } T} p=\operatorname{ContT}(\lambda k \rightarrow p \gg k) \\
& \text { exit' :: ContPipe' } i \text { o a } \\
& \text { exit }{ }^{\prime}=\text { abort }\left(\text { lift }_{\text {ReaderT }}\left(\text { lift }_{\text {ReaderT }}(\text { return }())\right)\right) \\
& \text { effect }{ }^{\prime}:: \text { IO } a \rightarrow \text { ContPipe' }^{\prime} \text { i o a } \\
& \text { effect }{ }^{\prime} e=\text { lift }_{\text {ContT }}\left(\text { lift }_{\text {ReaderT }}\left(\text { lift }_{\text {ReaderT }} e\right)\right)
\end{aligned}
$$

\section{Bidirectional Pipes}

So far we have covered unidirectional pipes where information flows in one direction through the pipe, from the output operations in one pipe to the input operations in the next pipe downstream. However, some use cases also require information to flow upstream and pipes that support this are called bidirectional.

The Proxy data type at the core of the pipes library [?] implements bidirectional pipes. The operations request and respond are respectively downstream and upstream combinations of input and output. In addition, Proxy is also a monad transformer that embed effects of monad $m$.

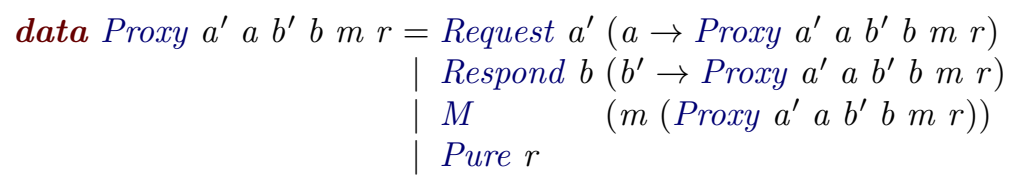

We refer to the pipes source code [?] for the implementation of the corresponding merge $_{P L}$ and merge $_{P R}$ functions, which are called $+\gg$ and $\gg \sim$.

We obtain a more efficient function-based representation by adapting the derivation of Sections ?? and ??. This yields the parameter type PCPar.

newtype PCPar i o a $=$ PCPar $(o \rightarrow$ PCPar o $i a \rightarrow a)$

The Result $t_{R}$ counterpart for Proxy takes two such PCPars as input. In addition, the result type $r$ is now a monadic type $m r$ to be able to lift operations once it is wrapped with $C$ ont.

$$
\begin{gathered}
\text { type ProxyRep } a^{\prime} \text { a } b^{\prime} b m=\forall r . P C P a r a a^{\prime}(m r) \rightarrow \quad \text {-- request } \\
\text { PCPar } b^{\prime} b(m r) \rightarrow \quad \text {-- respond } \\
m r
\end{gathered}
$$

Then, we can proceed with defining the merge function for ProxyRep and the Cont-wrapped version similar to Result $_{R}$. 


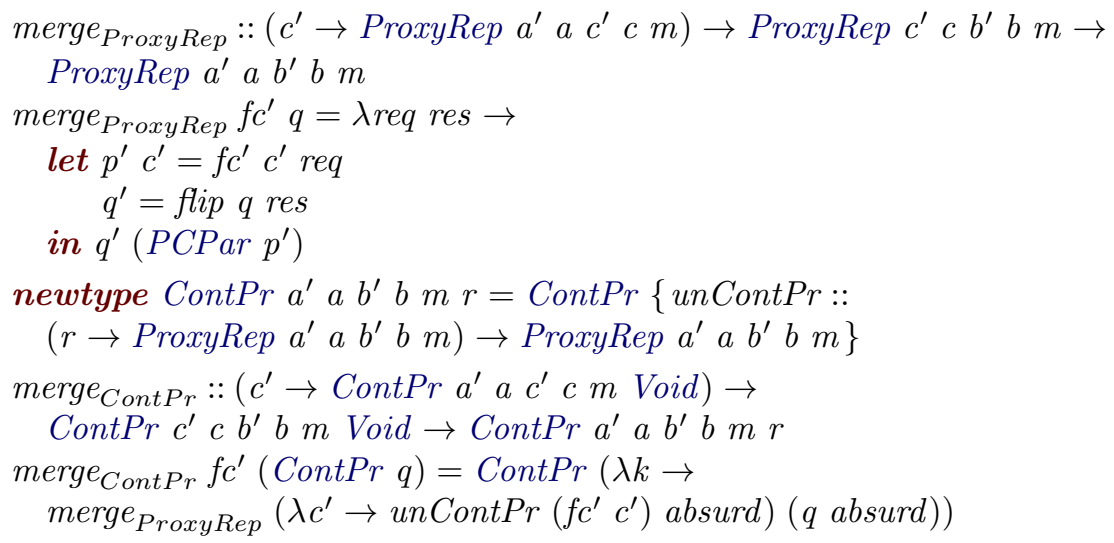

\section{Benchmarks}

Figure ?? shows the results of Spivey's primes benchmark, which calculates the first $n$ primes. The benchmarks are executed using the criterion library [?] on an Intel Core i7-6600U at $2.60 \mathrm{GHz}$ with $8 \mathrm{~GB}$ memory running Ubuntu 16.04 and GHC 8.4.3, with -02 enabled. $^{2}$

The figure compares the pipes (v4.3.9) and conduit (v1.3.0.3) libraries to Spivey's original implementation (contpipe) and our generalized form (proxyrep).

We can see that the former two libraries, which use an ADT representation, both show the quadratic performance behaviour for a use case with a high amount of merge steps. On the other hand, the latter two show the improved performance behaviour. The slight overhead of proxyrep compared to contpipe can be explained by the specialization to $I O()$ in the latter type.

The appendix contains the results of some additional microbenchmarks.

\section{Related Work}

We have covered the main related works of Spivey [?], Shivers and Might [?] and the pipes library [?] in the body of the paper. Below we discuss some additional related work.

Encodings The Church [?,?] and Scott [?] encodings encode ADTs using functions. The encoding derived in this paper has a close connection to the Scott encoding. The Scott encoding for Producer and Consumer are ScottP and ScottC. By moving the quantified variable $a$ to the definition, we obtain $S P$ and $S C$.

$$
\begin{aligned}
& \text { newtype } S \operatorname{cott} P o=S \operatorname{cott} P(\forall a \cdot(o \rightarrow S \operatorname{cott} P o \rightarrow a) \rightarrow a) \\
& \text { newtype } S \operatorname{cott} C i=S \operatorname{cott} C(\forall a \cdot((i \rightarrow S \operatorname{cott} C i) \rightarrow a) \rightarrow a)
\end{aligned}
$$

\footnotetext{
${ }^{2}$ The benchmarks are at https://github.com/rubenpieters/orth-pipes-bench.
} 


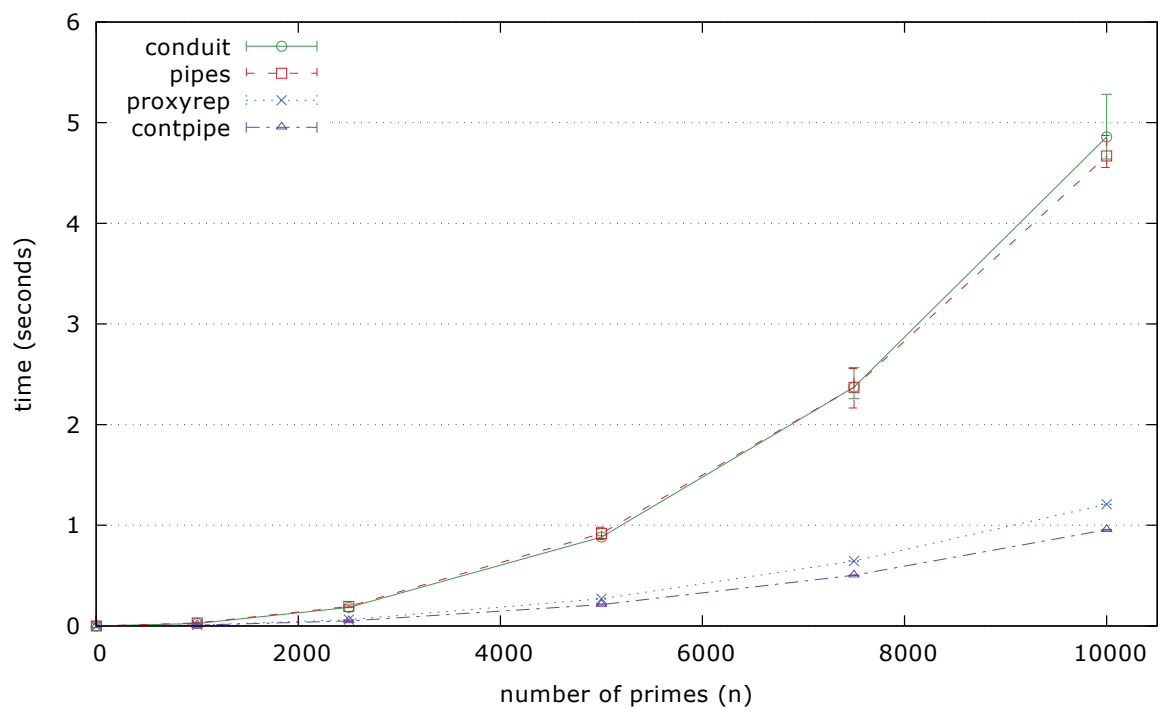

Fig. 1. Results of the primes benchmark.

$$
\begin{aligned}
& \text { newtype } S P \text { o } a=S P((o \rightarrow S P \text { o } a \rightarrow a) \rightarrow a) \\
& \text { newtype } S C \text { i } a=S C(((i \rightarrow S C \text { i } a) \rightarrow a) \rightarrow a)
\end{aligned}
$$

Then, $\forall a . S P$ o $a$ is representationally equivalent to Producer $_{A l t}$ and similarly for $\forall a . S C i$ a and Consumer $_{A l t}$ (see the appendix).

If we look at the Scott encoding ScottPipe $e_{\infty}$ for Pipe $_{\infty}$, we can obtain an equivalent representation to Result $_{R}$ by using $S P$ and $S C$ instead of $S \operatorname{cottPipe} e_{\infty}$ in the parameter corresponding to their operations.

$$
\begin{aligned}
& \text { newtype } \text { ScottPipe }_{\infty} \text { i o }=\text { ScottPipe }_{\infty} \\
& \quad\left(\forall a .\left(o \rightarrow \text { ScottPipe }_{\infty} i o \rightarrow a\right) \rightarrow\left(\left(i \rightarrow \text { ScottPipe }_{\infty} i o\right) \rightarrow a\right) \rightarrow a\right) \\
& \text { type } S P_{\infty} i o=\forall a .(o \rightarrow S P \text { o } a \rightarrow a) \rightarrow((i \rightarrow S C i a) \rightarrow a) \rightarrow a
\end{aligned}
$$

We dubbed this the orthogonal encoding due to the separation of the operations.

Conduit The conduit library [?] is another popular choice for Haskell stream processing. The two main differing points of conduit with pipes is a builtin representation of leftovers and detection of upstream finalization. Leftovers are operations representing unprocessed outputs. For example in a takeWhile pipe, which takes outputs untill a condition is matched, the first element not matching the condition will also be consumed. This element can then be emitted as a leftover, which will be consumed by the downstream with priority. Detecting upstream finalization is handled by input returning Maybe values, where Nothing represents the finalization of the upstream. 
Parsers Spivey mentioned in his work [?] that the ContPipe approach might be adapted to fit the use case of parallel parsers [?]. However, after gaining more insight into ContPipe, it does not seem that the merging operation for parsers immediately fits the pattern presented in this paper. One of the problematic elements is the fail operation, which is not passed as-is to the newly merged structure, but given a non-trivial interpretation. Namely, an interpretation dependent on the other structure during the recursive merge process.

Shallow To Deep Handlers The handlers framework by Kammar et al. [?] supports both shallow handlers, based on case analysis, and deep handlers, based on folds. They cover an example of transforming a producer and consumer merging function from shallow handlers to deep handlers. This example is related to our simplified setting in Section ??. To do this they introduce Prod and Cons, which are equivalent to our ProdPar and ConsPar. Compared to their example, we take a more step-by-step explanatory approach and additionally move to more complicated settings in our further sections.

Multihandlers The Frank language [?] is based on shallow handlers and supports a feature called multihandlers. These handlers operate on multiple inputs which have uninterpreted operations, much like pattern matching on multiple free structures. The patterns we have handled in this paper are concerned with pattern matching on multiple data structures and a mutual relation between these functions. This seems like an interesting connection to investigate further.

\section{Conclusion}

We have given an in-depth explanation of the principles behind the fast merging of the three-continuation approach. We have given a series of steps to derive this fast implementation from the less efficient one.

We apply this pattern to the setting of bidirectional pipes, as in the pipes library. This results in a more general version of this representation, but still has the same performance due to its efficient merge implementation.

We apply Spivey's benchmarks [?] to check that our generalized encoding retains similar performance. We also include the pipes library in the benchmark to compare with a commonly used implementation of bidirectional pipes.

This pipes encoding has been made available as a library on github ${ }^{3}$.

\section{Acknowledgements}

We would like to thank Nicolas Wu, Alexander Vandenbroucke and the anonymous PADL reviewers for their feedback. This work was partly funded by the Flemish Fund for Scientific Research (FWO).

\footnotetext{
${ }^{3}$ https://github.com/rubenpieters/Orthogonal-Pipes
} 